\title{
PABELLÓN DE GRAN BRETAÑA EN LA EXPO-92 DE SEVILLA-ESPAÑA
}

\author{
(THE BRITISH PAVILION IN THE EXPO'92 WORLD FAIR IN SEVILLE - SPAIN)
}

Nicholas Grimshow \& Partners

Fecha de recepción: 13-1-92

\section{RESUMEN}

Se exponen en estas páginas la composición y funcionamiento de la fachada acristalada del pabellón de Gran Bretaña en la EXPO'92 de Sevilla.

Un gran paño de $70 \times 18 \mathrm{~m}$ que sirve de base para una gran cascada de agua, que temple el calor sevillano.

Las piezas de vidrio son de $2,5 \times 1,8 \mathrm{~m}$ de tamaño, con un espesor de $12 \mathrm{~mm}$ y una pequeña trama de puntos en su superficie del $20 \%$. Se utiliza la silicona estructural como su único elemento de unión.

La estructura colgante, a la que se anclan los paneles, es reticular y se compone de:

Tirantes verticales de $8 \mathrm{~mm}$ de diámetro, perfiles horizontales de aluminio, las piezas de anclaje en acero inoxidable y las de ajuste en la parte superior.

También se destaca en esta fachada la integración de todo el sistema de tuberias y conductos necesarios para el funcionamiento de la cascada.

\section{SUMMARY}

This article contains the description of the composition and functioning of the glazed façade of the British pavilion in the EXPO'92 World Fair in Seville.

A panel of $70 \times 18 \mathrm{~m}$ serves as a base for a large waterfall which will soothe the heat of Seville.

The dimensions of the glass pieces are $2,5 \times 1,8 \mathrm{~m}$ and they are $12 \mathrm{~mm}$ thick with a small spotted weft occupying $20 \%$ of the surface. The structural silicone is the only unifying element used.

The hanging structure, to which the panels are tied, is reticulate and is composed of:

Vertical ties of $8 \mathrm{~mm}$ of diameter, horizontal aluminium profiles, bracing pieces made of stainless stell and fitting pieces in the upper part.

Another prominent feature of this façade is the integration of the whole system of pipework and tubes necessary for the functioning of the waterfall.

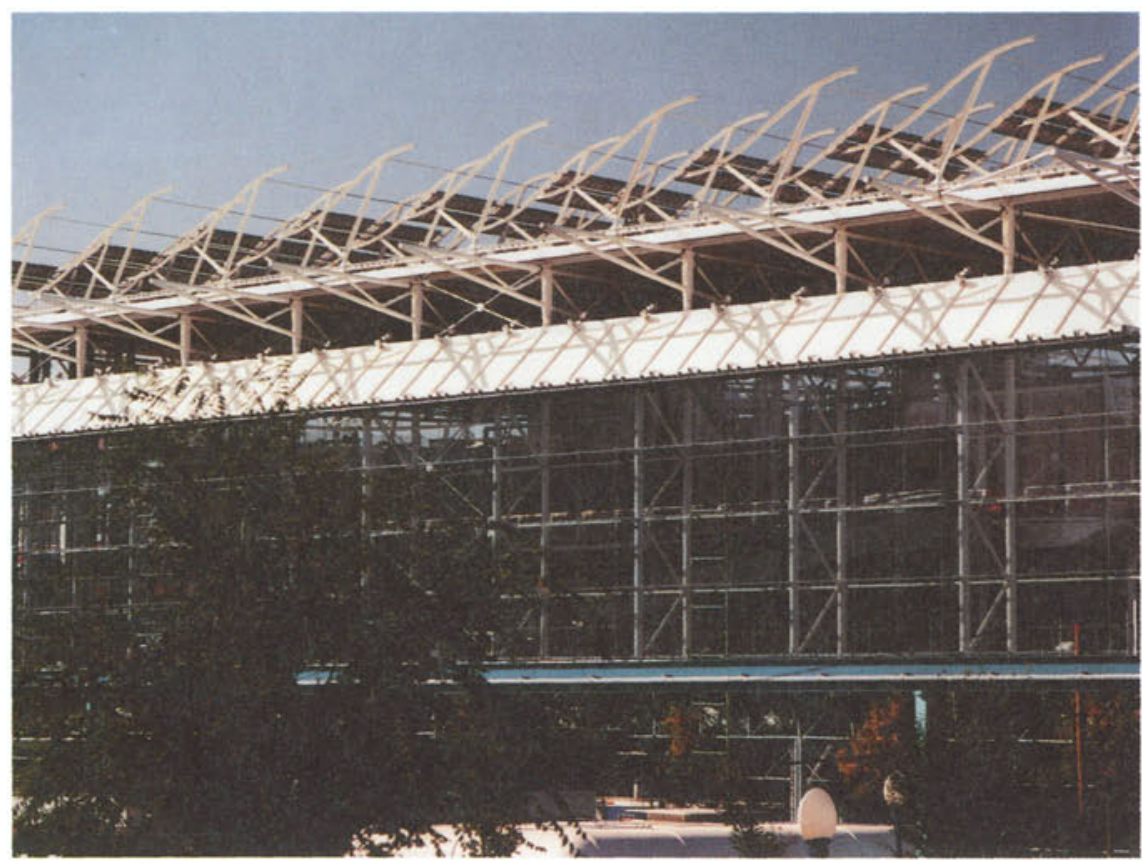




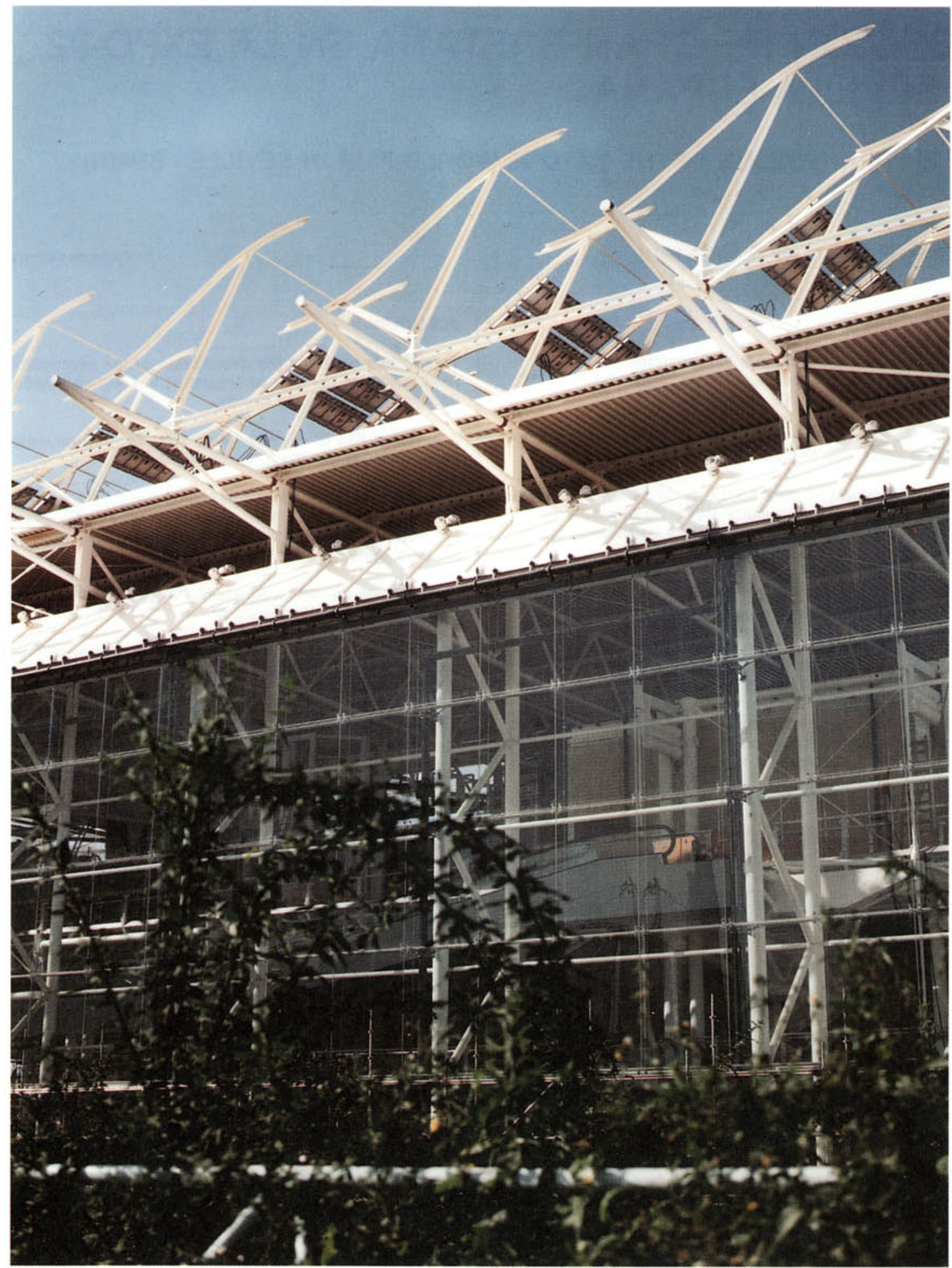




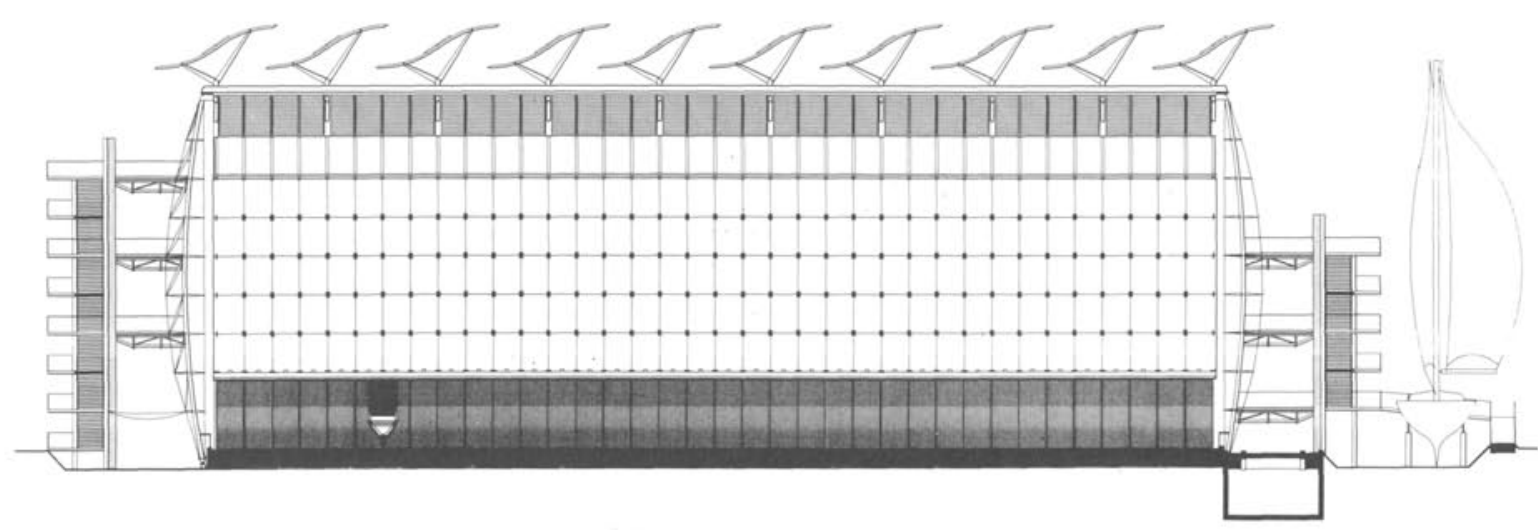

ALZADO ESTE

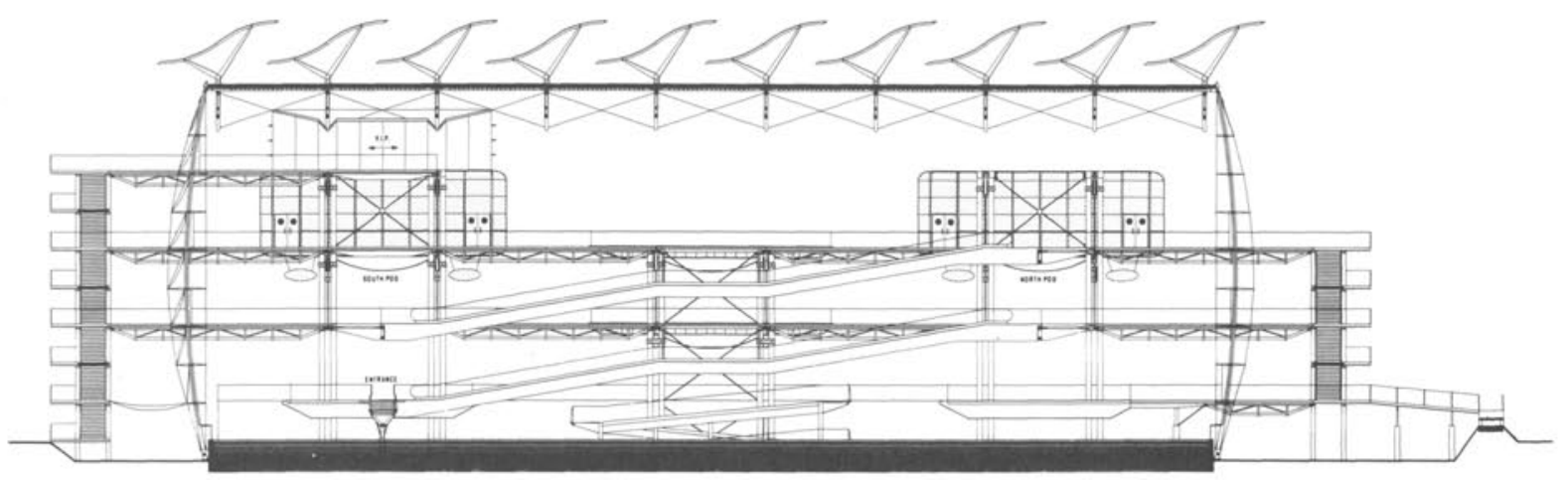

SECCIÓN LONGITUDINAL

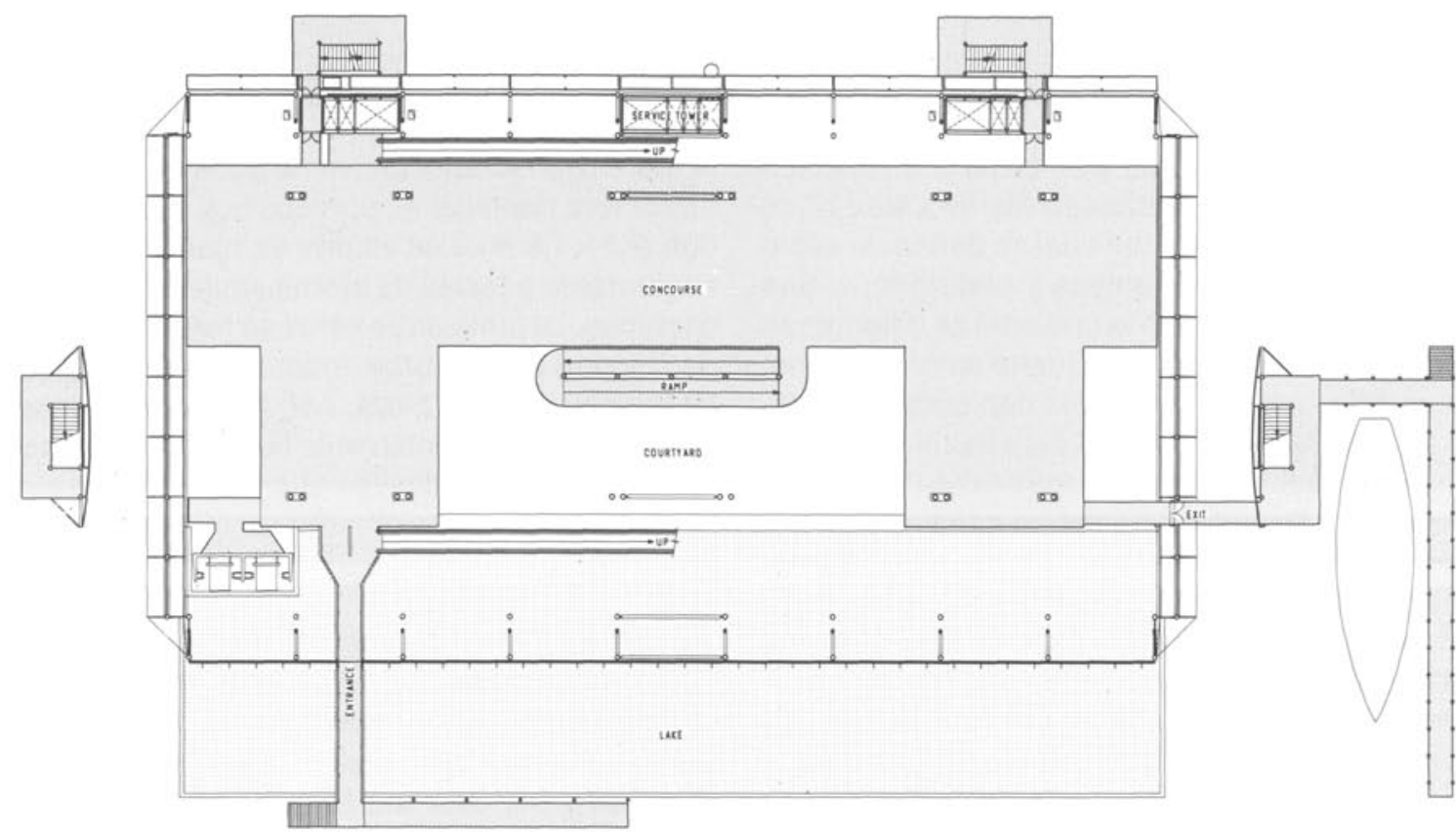

PLANTA BAJA 


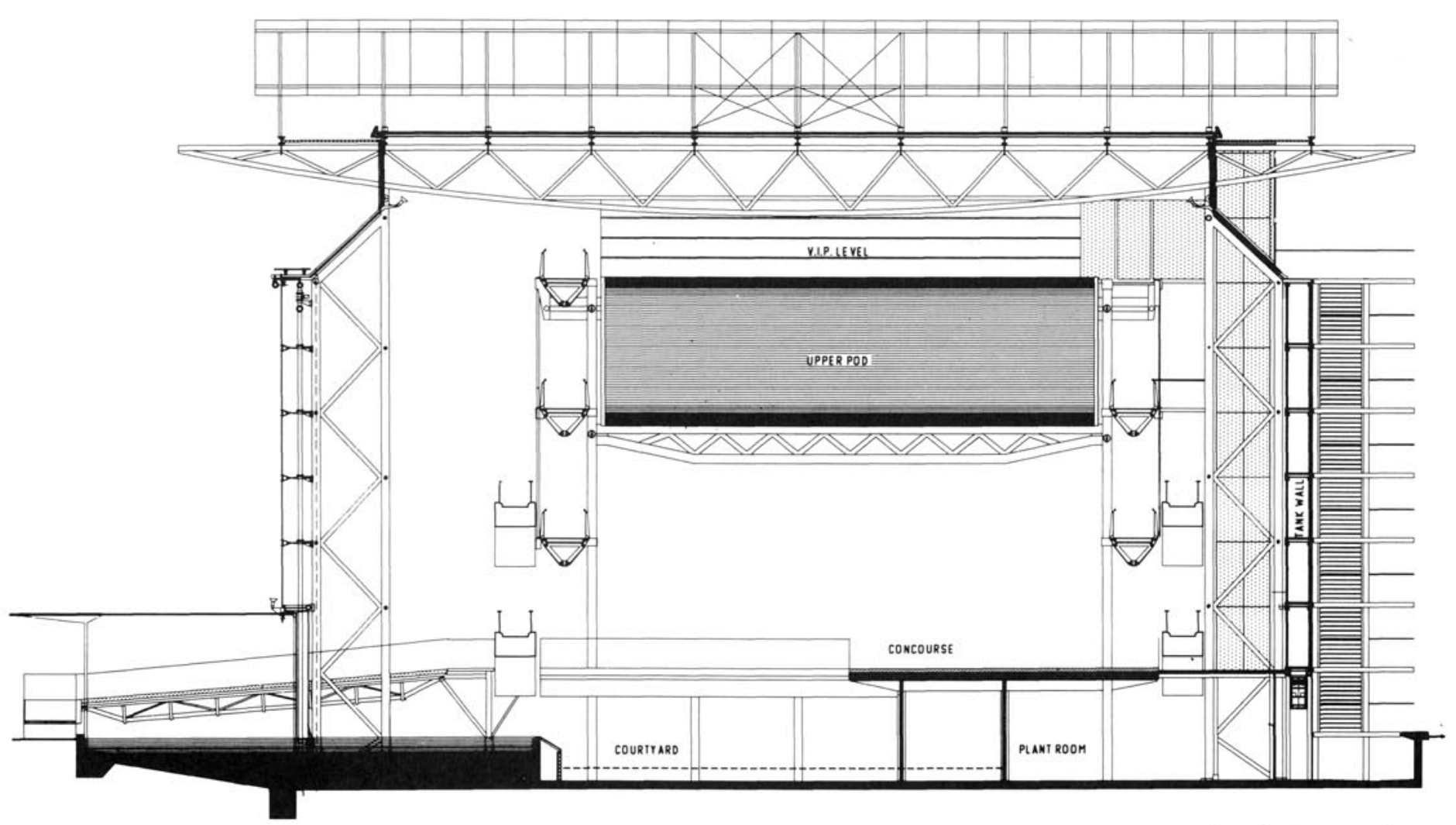

Sección transversal.

Edificio de vidrio y acero, el Pabellón de Gran Bretaña es un gran contenedor porticado (de $80 \times 40 \times 27 \mathrm{~m}$ ) abierto y continuo, dentro del cual se define un espacio fluido entre terrazas, rampas y plataformas. Sus cinco fachadas (incluyendo la cubierta) se determinan en función de su adaptación al fuerte calor sevillano. En la cubierta, grandes parasoles dan sombra al plano del techo, a la vez que sirven para instalar paneles solares. En la fachada Oeste, la más caliente, un gran muro trombe a base de tanques de agua absorbe el calor recibido. En las fachadas Sur y Norte grandes lamas horizontales graduan el paso de la luz a modo de persianas. Y en la fachada Este (la cual nos ocupa), una gran pared de agua servirá para templar la radiación solar que la atraviesa, a la vez que nos ofrece una impresionante fachada principal (de $70 \times 18 \mathrm{~m}$ ), a través de la cual se accede al pabellón.

Esta pared de agua es soportada por una base vertical de vidrio. La base, en cuestión, se construye como un cerramiento colgado compuesto en piezas de (C) Consejo Superior de Investigaciones Científicas Licencia Creative Commons 3.0 España (by-nc) vidrio, cuya modulación viene dada en función de la estructura principal de pórticos que sustenta el pabeIlón $(2,5 \times 1,8 \mathrm{~m})$. Los vidrios se fijan a su estructura sustentante a través de atornillamiento en sus cuatro esquinas. La junta entre éstos se resuelve con un tipo de silicona que absorbe, independientemente, las dilataciones de cada pieza. Así, nos encontramos ante una pared lo suficientemente lisa y continua como para permitir que se deslice el agua libremente por su superficie.

La estructura, de la que se cuelgan las piezas de vidrio, consiste fundamentalmente en una reticula ortogonal compuesta de elementos verticales y horizontales, con misiones y grados de trabajo muy distintos (Págs. 80 y 81). Diferencias que se ven manifestadas en su forma:

- delgados cables para los montantes (de $8 \mathrm{~mm}$ de espesor), que sólo trabajan verticalmente a trachttp://informesdelaconstruccion.revistas.csic.es 


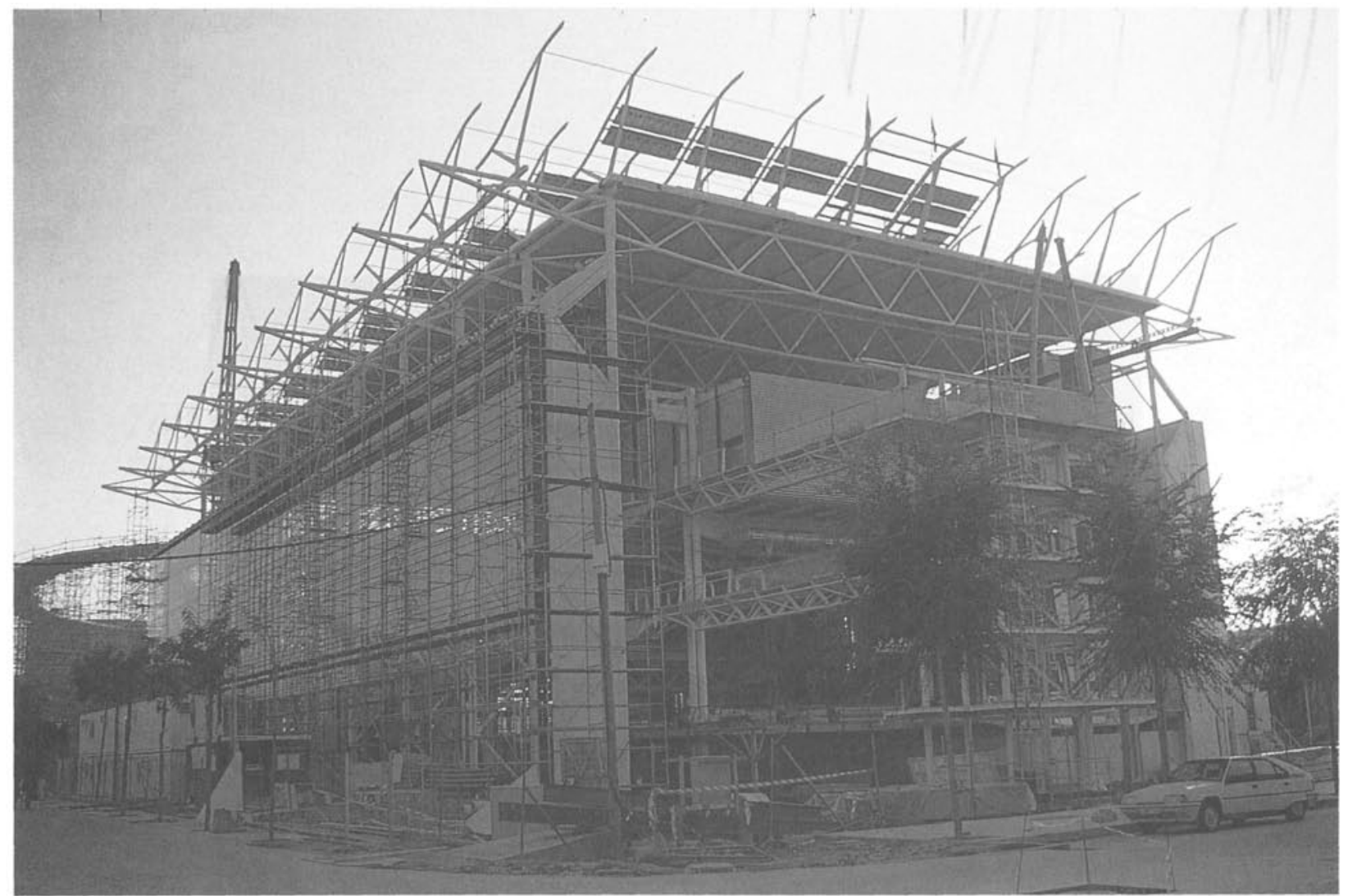

El pabellón en construcción.

ción, con sus piezas de ajuste para la tolerancia vertical;

- estrechos perfiles de aluminio horizontales separados del vidrio que, con su forma, dan rigidez a la fachada contra el viento, a la vez que ofrecen un carril continuo para el replanteo horizontal de las piezas de fijación al vidrio;

- cada una de las piezas de fijación, en acero inoxidable, recoge dos esquinas de las lunas adyacentes, y ajusta su replanteo planar respecto a la dirección perpendicular a la fachada.

Aparte de la construcción de esta base de vidrio, la instalación necesaria para el funcionamiento de la cascada se hace fundamental para imbricar ambos sistemas.

A través de cinco bombas de agua, que funcionan por energía solar, se recicla el agua de la cascada. Estas cinco bombas de agua responden a otros tantos conductos verticales, que la estructura colgada del cerramiento debe salvar coincidiendo con cada pórtico.

Una vez en la parte superior del cerramiento, todo el sistema de tubos, pipetas y sifones condiciona la solución constructiva de este remate, dando lugar a una "peculiar cornisa".

El vidrio empleado (de $12 \mathrm{~mm}$ de espesor), contempla el factor de seguridad y transparencia como primordiales. Por tanto, como aislamiento, en vez.de acudir a la cámara de climalit, o a alguna de las propiedades reflectantes de las últimas generaciones de vidrio, que alteraría la transparencia deseada, se opta por la solución de motear la superficie con una sutil trama ortogonal de pequeños puntos oscuros (inapreciables a más de $2 \mathrm{~m}$ de distancia) consiguiendo así un leve filtro solar proporcional al \% de la trama, y que permite la transparencia deseada.

http://informesdelaconstruccion.revistas.csic.es 

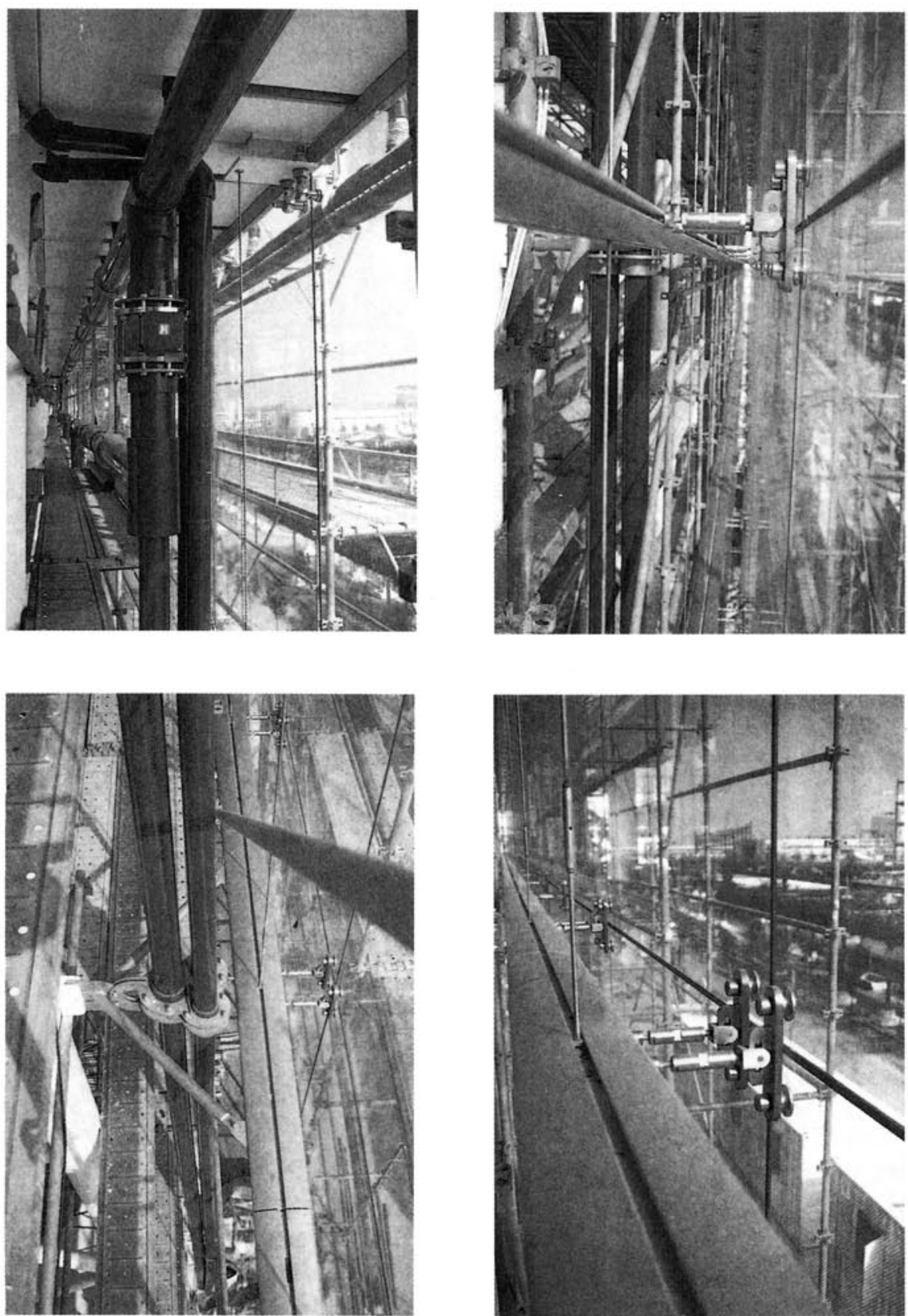


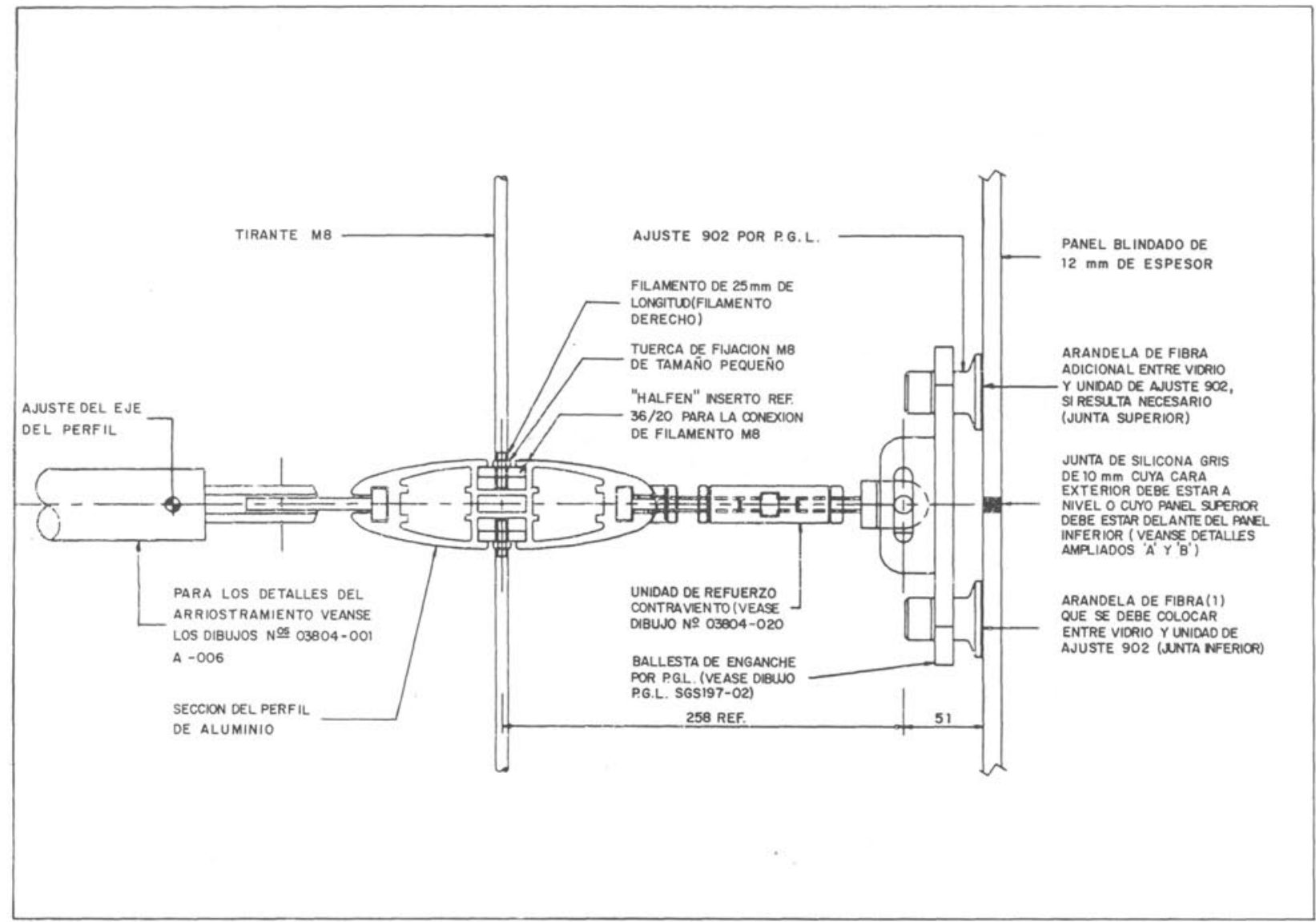

Detalle de la fijación del vidrio y de la estructura de sustentación.

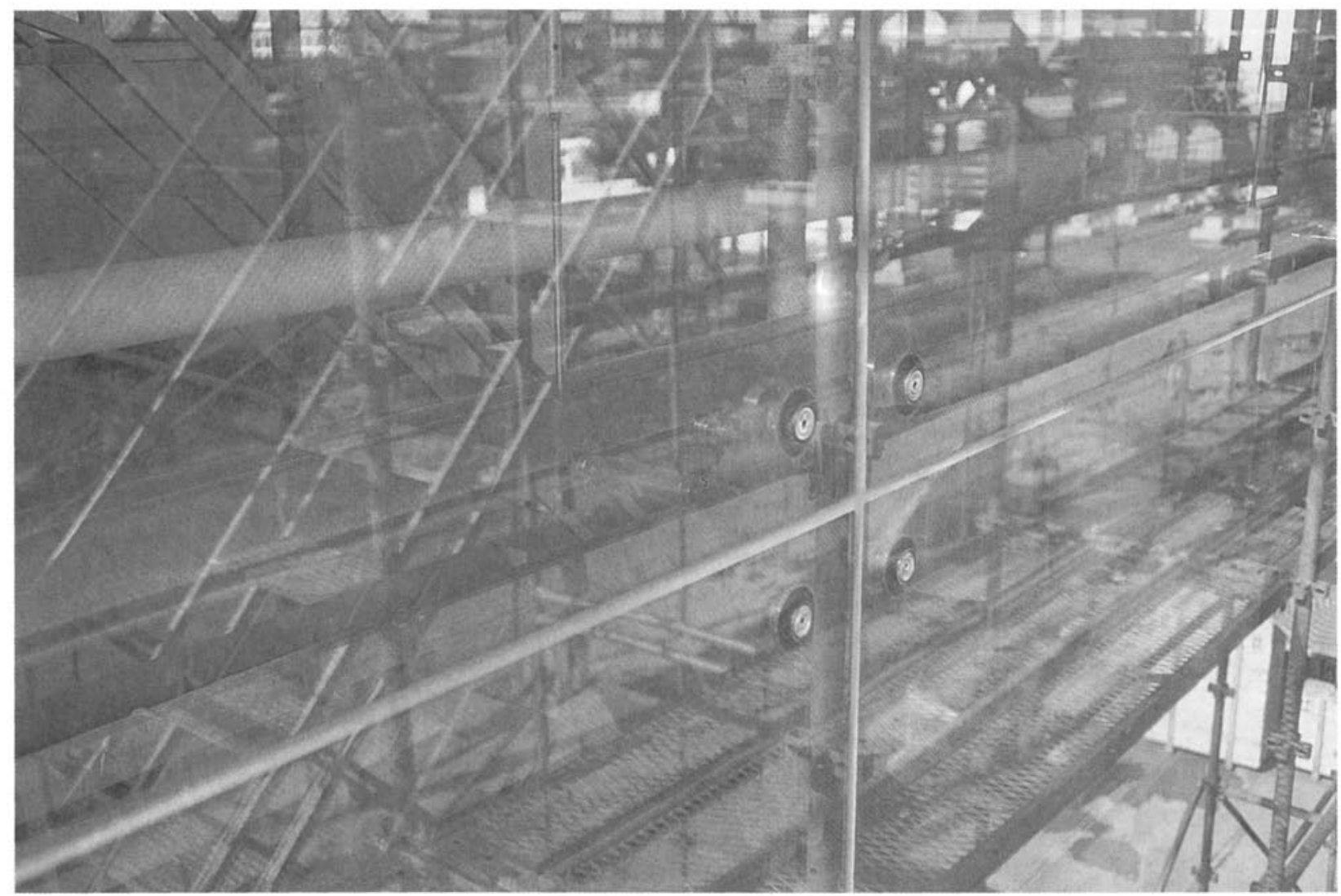

Encuentro de cuatro paneles desde el exterior. 

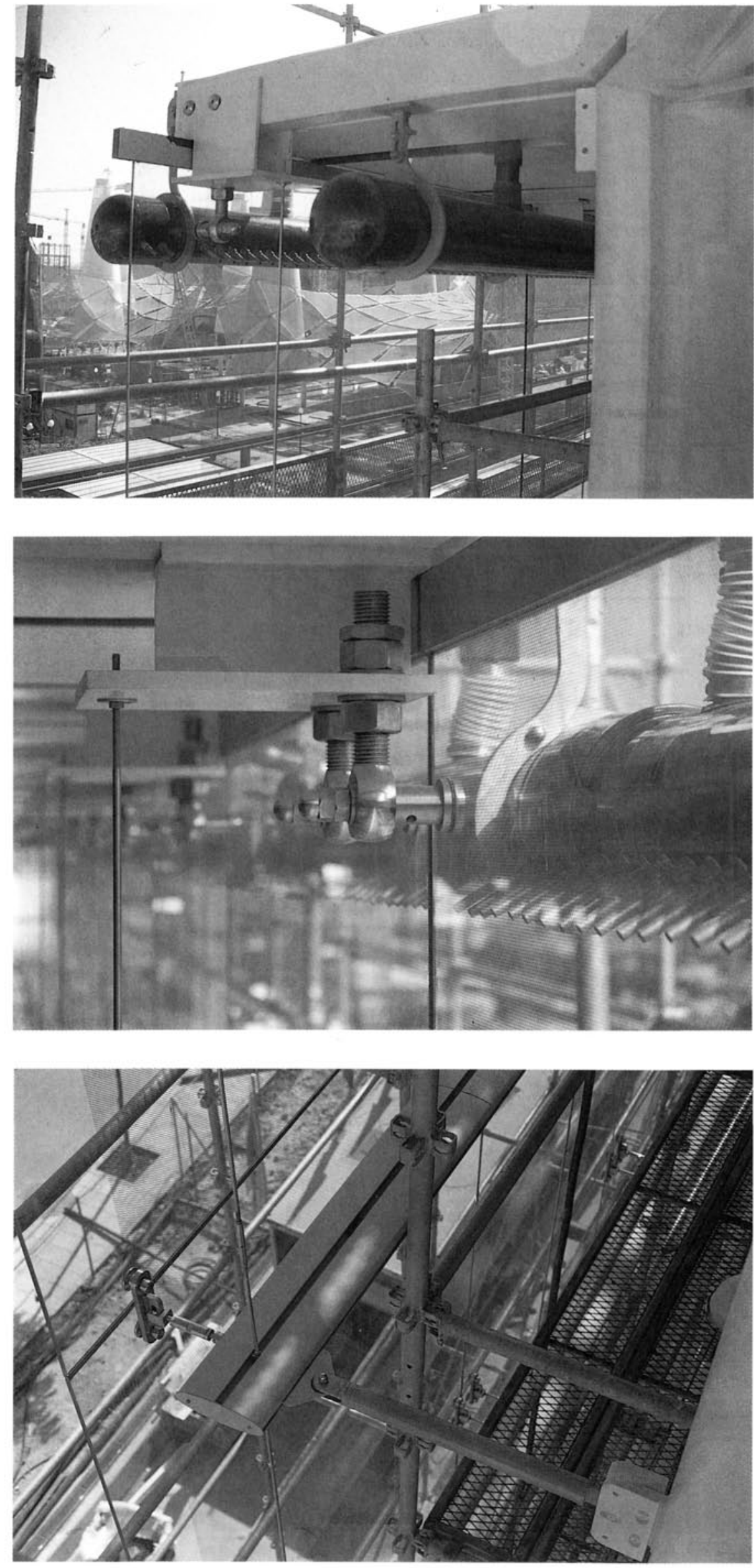

(c) Consejo Superior de Investigaciones Científicas Licencia Creative Commons 3.0 España (by-nc)
Extremo lateral de la pared de cristal. Se aprecia el perfil horizontal de alu. minio para las acciones horizontales y la biela de transmisión a la estructu. ra principal.

La pared en su parte superior. Se aprecian las ró. tulas superiores para fijación del vidrio El sis. tema de anclaje de los tirantes verticales y los sifones de impulsión de agua en el exterior.

Extremo lateral de la pa. red de cristal a la altura de la cornisa. Se aprecian las piezas para el cuelgue de la estructura y el rema. te de los tubos para el agua.

http://informesdelaconstruccion.revistas.csic.es 
